\title{
Pancreas Transplant Salvage by Proximal Loop Ileostomy and Distal Ileostotomy Tube for Duodenal Stump Leak after Enteric Conversion
}

\author{
Monroy-Cuadros Mauricio, Rodriguez-Velez Cesar \\ Division of Transplantation, Department of Surgery, Foothills Medical Centre, \\ Calgary, AB, Canada \\ E-mail:mauricio.monroy@albertahealthservices.ca \\ Received August 26, 2010; revised October 30, 2010; accept November 20, 2010
}

\begin{abstract}
After pancreas transplantation, some patients with bladder drainage (BD) of the pancreatic duct will need to be converted to enteric drainage (ED) because of reflux pancreatitis, metabolic acidosis, and urological complications. However, ED is associated with higher rates of duodenal stump leak, intra-abdominal abscess, and peritonitis. In some cases of enteric anastomosis leakage, a primary repair can be attempted, but in more severe cases, graft pancreatectomy is indicated. We report one patient who received a combined kidney and pancreas transplant with BD of exocrine secretions, but who required ED conversion 6 years later because of persistent metabolic acidosis and adverse urological symptoms. However, a significant duodenal leak was discovered 4 days post-operatively. To salvage the transplanted pancreas, we performed a diverting loop ileostomy proximal to the entero-entero anastomosis and the distal section was drained retrogradely with an ileostostomy tube, allowing the area of the leak to heal. Three months later, the ileostomy was reversed without complications, the symptoms that led to the ED conversion resolved, and the kidney and pancreas allografts remain functional 48 months later. We suggest that this might be a method by which transplanted pancreas may be salvaged in the case of leakage after ED conversion.
\end{abstract}

Keywords: Pancreas, Transplant, Conversion leak

\section{Introduction}

The history of pancreas transplantation has been marked by surgical complications. In the early era of pancreas transplantation, $25 \%$ of the grafts were lost because of technical issues [1]. Factors such as advances in preservation techniques, refinements in immunosuppressive regimens, and the development of surgical techniques have improved the results and outcomes of the pancreas transplant. However, pancreas transplantation still has a higher rate of complications than other organ transplants, including thrombosis, rejection, surgical complications [2], infections, and urologic complications [3]. Management of pancreatic exocrine secretions has been a matter of debate for years, and a variety of techniques have been proposed and abandoned. For decades, bladder drainage (BD) was the most common method of duct management; it has the advantage of fewer episodes of sepsis while providing a means to monitor episodes of rejection through the measure of urinary amylase [4]. However, $\mathrm{BD}$ is not a physiologic procedure and is associated with high morbidity including reflux pancreatitis, metabolic acidosis, and urological complications [5-7] that are becoming the most common indication for conversion to enteric drainage (ED) [3,5-7]. Up to $25 \%$ of patients with $\mathrm{BD}$ will need conversion to ED within 10 years of the transplant $[3,6,8]$. ED is a more physiologic technique with lower incidence of urological complications but is associated with higher rates of duodenal stump leak, intra-abdominal abscess, and peritonitis $[6,9,10]$; the rate of surgical complications after ED conversion is reported to be $10 \%$ to $20 \%[3,6,11]$. Although major leaks from the enteric anastomosis only occur in $5 \%$ to $7 \%$ of patients [10-12], they can represent a high rate of pancreatic graft loss $[13,14]$. In cases of a circumscribed leak, a primary repair can be attempted, but in more severe cases with significant leak, graft pancreatectomy is indicated in almost $95 \%$ of patients [11]. 
We herein report one patient who developed a significant duodenal leak after pancreas transplant ED conversion that was salvaged with a pancreas transplant exclusion via a proximal loop ileostomy and distal ileostostomy tube. No postoperative complications were recorded, and the patient remains insulin-independent 48 months after the conversion.

\section{Methods and Results}

The patient is a Caucasian male, who at the age of 14 was diagnosed with type 1 diabetes mellitus, and by the age of 47 had reached Chronic Renal Failure Stage V requiring dialysis. The patient received a combined kidney and pancreas transplant with BD of exocrine secretions. Both kidney and pancreas allografts had good renal and pancreatic function immediately after surgery. The patient received induction with polyclonal antibodies. Immunosuppression was maintained with triple therapy including FK 506, Mycophenolate Mofetil, and steroids. During the first 18 months after the transplant, the patient had three biopsy-proven acute rejection episodes that clinically involved the renal allograft alone. The first two rejections were reversed with the use of anti-lymphocyte globulin and the third episode with the use of steroids.

At 4 years after transplantation, the patient presented with hematuria on several occasions. Cystoscopy showed only inflammation and erythema of the urethra. At 6 years after transplantation, the patient had persistent metabolic acidosis and urological symptoms including severe dysuria and intractable burning pain requiring rehydration, sodium bicarbonate replacement, and antibiotics on a more regular than repetitive basis. An ED conversion was performed without intra-operative complications.

On post-operative day 4 after the ED conversion, the patient presented with abdominal pain and distention, and a drainage of intestinal contents was noted through surgical wound. Exploratory laparotomy found a significant duodenal leak with contamination of the abdominal cavity. The pancreas had a normal appearance, but the duodenal segment was viable, edematous, and fragile. A primary closure was attempted, but as the tissues were inflamed and fragile, a patch of small bowel was use to cover the anastomosis disruption. We decided to exclude the pancreas transplant with a diverting loop ileostomy proximal to the entero-entero anastomosis with the distal section drained retrogradely with an ileostostomy tube and drains around the entero-entero anastomosis (Figure 1). During the post-operative time, intestinal transit was reduced with the use of parenteral nutrition and Octreatodide. The postoperative course was uneventful and the

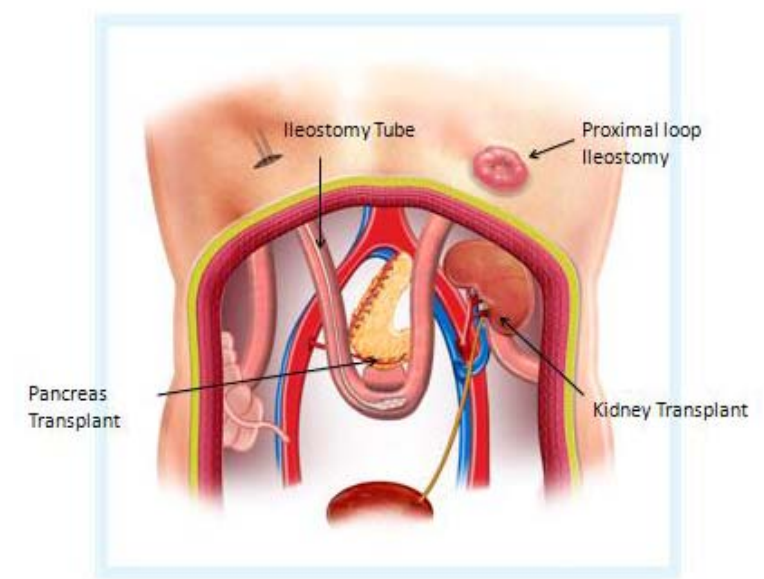

Figure 1. Diagram of the proximal diverting loop ileostomy and distal ileostomy tube to defunctionalize the pancreas transplant. The small bowel content was drained through the ileostomy, and the exocrine secretion of the pancreatic allograft was collected with the ileostomy tube, creating a controlled fistula.

patient was discharged home. A contrast enema was done with no evidence of strictures distal or proximal to the ileostomy. Three months later, the ileostomy was reversed without complications.

After the ED conversion, the symptoms that indicated the conversion resolved, both kidney and pancreas allografts remain functional at 48 months after the procedure, and the patient remains insulin-independent.

\section{Discussion}

The vast majority of pancreas transplants are performed with ED of the exocrine secretions; however, approximately $20 \%$ of transplant programs continue to report the use of BD. Metabolic acidosis, recurrent urinary tract infections, and reflux pancreatitis are the most common indications for conversion from $\mathrm{BD}$ to $\mathrm{ED}[6,13]$; however, enteric leaks are a risk after ED. Some authors report that in cases of circumscribed duodenoenteric leakage, a primary repair of the leak can be attempted. However when moderate or severe peritonitis and sepsis is manifested, graft pancreatectomy is the best option [6].

Several methods have been reported for rescue of the transplant after enteric leakage. In 2003, Orsenigo et al. described a surgical technique for management of a duodenojejunostomy leakage in four patients by occluding the main pancreatic duct with a synthetic polymer injection [15]. In 2009, Ablorsu et al. reported a case where the graft duodenum was removed and the main pancreatic duct was anastomosed to the bladder [16]. In 2010, Boggi et al. reported a total duodenectomy with enteric duct drainage for the management of duodenal complica- 
tions occurring after pancreas transplantation [17]. The case history described here is unique in several aspects. In the case reported by Ablorsu, there was a necrosis of the duodenal segment after reperfusion of the pancreas, and a duodenectomy with BD was performed; preservation or reperfusion injuries and edema of the duodenal stump were important factors that led to this treatment choice. In our case, the perfusion of the graft was not an issue, but the important factors were that the patient was already receiving immunosuppression therapy and the presence of an intra-abdominal abscess may have had a high risk for morbidity and mortality. The intraoperative findings suggested that a primary closure or reanastomosis of the duodenal stump was not a good option given the degree of inflammation, edema, and contamination with intestinal contents; however the condition of the duodenal stump was appropriate and did not require a duodenectomy as reported by Ablorsu and Boggi.

Our decision to exclude the pancreas transplant was mainly based on good results of the pyloric exclusion technique used on the management of pancreatico-duodenal complex injuries [18]. Our decision to achieve pancreatic exclusion through a diverting loop proximal ileostomy with a distal retrograde ileostostomy tube gave us the opportunity to divert the intestinal contents through the ileostomy, allowing the area of the leak to heal. The exocrine secretions from the pancreas were collected by the distal ileostomy tube, creating a controlled pancreatic fistula. To our knowledge, this is the first time this procedure has been used for this circumstance. We suggest that proximal diverting loop ileostomy with distal ileostomy tube insertion might be a method by which transplanted pancreas may be salvaged in the case of leakage after ED conversion.

\section{References}

[1] R. W. Gruessner and D. E. Sutherland, "Transplantation of the Pancreas,” New York, Birkhauser, 2004.

[2] R. W. Gruessner, D. L. Dunn, P. J. Tzardis, R. I. Nakhleh, J. S. Najarian and D. E. Sutherland, "Complications Occurring after Whole Organ Duodenopancreatic Transplantation: Relation to the Allograft Duodenal Segment,” Transplantation Proceedings 1990, Vol. 22, No. 2, pp. 578-579.

[3] H. W. Sollinger, T. M. Sasaki, A. M. D’Alessandro, S. J. Knechtle, J. D. Pirsch, M. Kalayoglu, et al, "Indications for Enteric Conversion after Pancreas Transplantation with Bladder Drainage,” Surgery, Vol. 112, No. 4, 1992, pp. 842-846, discussion 845-846.

[4] M. Prieto, W. Collins, M. H. Scott and R. A. Sells, "Method for Home Monitoring of Urinary Amylase after Pancreas Transplantation,” Diabetes, Vol. 38, Sup. 1, 1989, pp. 68-70.
[5] H. W. Sollinger, E. M. Messing, D. E. Eckhoff, J.D. Pirsch, A. M. D’Alessandro, M. Kalayoglu et al, "Urological Complications in 210 Consecutive Simultaneous Pancreas- kidney Transplants with Bladder Drainage," Annals of Surgery, Vol. 218, No. 4, 1993, pp. 561-568; Discussion, pp. 568-570.

[6] M. West, A. C. Gruessner, P. Metrakos, D. E. Sutherland, R. W. Gruessner, "Conversion from Bladder to Enteric Drainage after Pancreaticoduodenal Transplantations," Surgery, Vol. 124, No. 5, 1998, pp. 883-893.

[7] M. Monroy-Cuadros, A. Salazar, S. Yilmaz and K. McLaughlin, "Bladder vs Enteric Drainage in Simultaneous Pancreas-kidney Transplantation,” Nephrol Dial Transplant, Vol. 21, No. 2, 2006, pp. 483-487. doi:10.1093/ndt/gfi252

[8] S. A. White, J. A. Shaw and D. E. Sutherland, "Pancreas transplantation,” Lancet, Vol. 373, No. 9677, 2009, pp. 1808-1817. doi:10.1016/S0140-6736(09)60609-7

[9] H. W. Sollinger, J. S. Odorico, S. J. Knechtle, A. M. D’Alessandro, M. Kalayoglu and J. D. Pirsch, "Experience with 500 Simultaneous Pancreas-kidney Transplants," Annnals of Surgery, Vol. 228, No. 3, 1998, pp. 284-296. doi:10.1097/00000658-199809000-00002

[10] D. E. Sutherland, R. W. Gruessner, D. L. Dunn, A. J. Matas, A. Humar, R. Kandaswamy, et al. "Lessons Learned from more than 1,000 Pancreas Transplants at a Single Institution,” Annals of Surgery, Vol. 233, No. 4 2001, pp. 463-501. doi:10.1097/00000658-200104000-00003

[11] C. Jimenez-Romero, A. Manrique, J. M. Morales, R. M. Lopez, E. Morales, F. Cambra et al. "Conversion from Bladder to Enteric Drainage for Complications after Pancreas Transplantation,” Transplantation Proceedings, Vol. 41, No. 6, 2009, pp. 2469-2471. doi:10.1016/j.transproceed.2009.06.077

[12] S. N. Wang, M. Sturdevant, R. Kandaswamy, R. G. Gruessner, D. E. Sutherland and A. Humar, "Technical Failure of the Pancreas after SPK Transplant: Are These Patients Good Candidates for Later Pancreas Retransplant?” Clinical Transplantation, Vol. 22, No. 1, 2008, pp. 50-54.

[13] P. van de Linde, P. J. van der Boog, A. G. Baranski, J. W. de Fijter, J. Ringers and A. F. Schaapherder "Pancreas Transplantation: Advantages of Both Enteric and Bladder Drainage Combined in a Two-step Approach,” Clinical Transplantation, Vol. 20, No. 2, 2006, pp. 253-257. doi:10.1111/j.1399-0012.2005.00477.x

[14] W. Steurer, H. Bonatti, P. Obrist, B. Spechtenhauser, R. Ladurner, W. Mark et al, "Incidence of Intraabdominal Infection in a Consecutive Series of 40 Enteric-drained Pancreas Transplants with FK506 and MMF Immunosuppression,” Transplant Intertional, Vol. 13, Sup. 1: 2000, pp. 195-198.

[15] E. Orsenigo, M. Cristallo, C. Socci, R. Castoldi, P. Fiorina, L. Invernizzi et al, "Successful Surgical Salvage of Pancreas Allograft,” Transplantation, Vol. 75, No. 2, 2003, pp. 233-236. doi:10.1097/01.TP.0000041784.27763.A9

[16] E. Ablorsu, S. Mehra, A. Tavakoli, T. Augustine, R. Pa- 
rarajasingam, "Staged Enteric Conversion after Duodenal Necrosis in Simultaneous Kidney and Pancreas Transplant from a Donor after Cardiac Death: A Case Report,” Transplantation Proceedings, Vol. 41, No. 5, 2009, pp. 1778-1780. doi:10.1016/j.transproceed.2009.02.081

[17] U. Boggi, F. Vistoli, M. Del Chiaro, C. Moretto, C. Croce, S. Signori et al. "Total Duodenectomy with Enteric Duct
Drainage: A Rescue Operation for Duodenal Complications Occurring after Pancreas Transplantation,” American Journal of Transplantation, Vol. 10, No. 3, 2010, pp. 692-697. doi:10.1111/j.1600-6143.2009.02981.x

[18] D. Feliciano, D. Mattox, E. Moore “Trauma,” 6th edition, New York, McGraw-Hill Professional, 2007. 